\title{
Production and hedging under correlated price and background risks
}

\author{
Kit Pong Wong ${ }^{1}$ (B)
}

Received: 23 November 2020 / Accepted: 5 October 2021 / Published online: 28 October 2021

(C) The Author(s), under exclusive licence to Associazione per la Matematica Applicata alle Scienze Economiche e Sociali (AMASES) 2021

\begin{abstract}
This paper examines the competitive firm that has to make its production and hedging decisions under correlated price and background risks. The background risk can be either financial or non-financial, which is accommodated by using a bivariate utility function. The separation theorem is shown to hold in that the firm's optimal output level depends neither on the firm's bivariate utility function nor on the joint distribution of the price and background risks. We derive necessary and sufficient conditions under which the firm optimally opts for an over-hedge (under-hedge). We further derive necessary and sufficient conditions under which hedging has positive (negative) effect on the firm's optimal output level. These conditions are shown to be related to the concept of expectation dependence and bivariate preferences that include correlation aversion (correlation loving) and cross-prudence (cross-imprudence).
\end{abstract}

Keywords Background risk · Forward hedging · Production

JEL Classifications D21 · D81 · G13

\section{Introduction}

Since the seminal work of Sandmo (1971), the theory of the competitive firm under price uncertainty has been extensively studied. One important strand of this literature is on the behavior of the firm when forward trading is allowed (Danthine 1978; Feder et al. 1980; Holthausen 1979), from which two notable results emanate. First, the separation theorem states that the firm's production decision depends neither on the

\footnotetext{
The author would like to thank Udo Broll and two anonymous referees for their helpful comments and suggestions. The usual disclaimer applies.
}

Kit Pong Wong

kitpongwong@hku.hk

1 Faculty of Business and Economics, University of Hong Kong, Pokfulam Road, Hong Kong, China 
firm's preferences nor on the underlying price distribution. Second, the full-hedging theorem states that the firm should completely eliminate its exposure to the price risk by adopting a full-hedge if the forward price is unbiased. ${ }^{1}$

Wong (2014) examines the robustness of the separation and full-hedging theorems when there are other sources of uncertainty that are aggregated into an additive and/or multiplicative background risk embedded in the firm's profit. The background risk as such is financial (risky investment income or random fixed costs) and is not necessarily independent of the price risk. Wong (2014) shows that the separation theorem is robust to the introduction of the correlated background risk, whereas the full-hedging theorem holds in the special case that the background risk is independent of the price risk. In the general case that the background risk is correlated with the price risk, Wong (2014) shows that the concept of expectation dependence (Wright 1987) plays a pivotal role in determining the firm's optimal forward position.

The purpose of this paper is to extend the model of Wong (2014) to the case wherein the background risk is allowed to be non-financial. A prominent example of non-financial background risk is political risk, which represents the uncertainty arising from government policies and actions that can have profound impacts on corporations. ${ }^{2}$ The term 'political risk' covers a variety of issues ranging from the threat of political violence to geopolitical tensions and exchange controls. ${ }^{3}$ Recent events of the global rise in protectionism and policy uncertainty in response to nationalist sentiments and economic security considerations, coupled with some immediate policy actions to tackle the COVID-19 pandemic, present new challenges that trigger great concerns around political risk. Another example of non-financial background risk is environmental risk that is associated with causing harm or damage to the environment. This is often related to a firm's core business. Certain industries, such as energy, are more likely than others to be subject to environmental litigation and regulatory intervention because of their potentially harmful impacts on the environment. ${ }^{4}$

To accommodate the non-financial nature of background risk, we use a bivariate utility function, $u(\pi, z)$, that defines over the firm's profit, $\pi$, and its exposure to the background risk, $z$. The firm is said to be correlation averse (correlation loving) if $\partial^{2} u(\pi, z) / \partial \pi \partial z<(>) 0$ and cross-prudent (cross-imprudent) in the background risk if $\partial^{3} u(\pi, z) / \partial \pi^{2} \partial z>(<) 0$ (Eeckhoudt et al. 2007). Correlation aversion (correlation loving) is characterized by preferences for combining good with bad (good with good or bad with bad), where the bad is a sure reduction in both the profit and the realization

\footnotetext{
1 The full-hedging theorem is analogous to a well-known result in the insurance literature that a risk-averse individual fully insures at an actuarially fair price (Mossin 1968).

2 The term 'political risk' is commonly used by the political risk insurance industry that offers a wide range of insurance products for non-commercial risks.

3 Based on computation linguistics, Hassan et al. (2019) develop a novel measure of firm-level political risk that is calculated as the share of a given firm's quarterly earnings conference calls devoted to political risk.

${ }^{4}$ Lu et al. (2021) propose to measure environmental risk at the industry level based on the Fama-French 48 industry classification that captures the characteristics of a firm's operations and inherent risks.
} 
of the background risk that lower the firm's utility (Eeckhoudt et al. 2007; Jokung 2011). ${ }^{5}$

We first show that the separation theorem holds in that the firm's optimal output level depends neither on the firm's bivariate utility function nor on the joint distribution of the price and background risks. Since the firm can always sell the last unit of its output forward, the marginal revenue is locked in at the predetermined forward price. As such, the usual optimality condition applies in that the marginal cost of production is equated to the known marginal revenue, which determines the firm's optimal output level.

We then derive necessary and sufficient conditions under which the firm optimally opts for an over-hedge (under-hedge). The full-hedging theorem holds if the background risk is independent of the price risk. When the firm is correlation averse (correlation loving), we show that the firm dislikes positive (negative) exposure to the residual price risk in the presence of the background risk if the two risks are positively expectation dependent. This gives rise to an additional hedging motive that induces the firm to sell more (less) of its output forward than a full-hedge so as to reduce the correlation risk, which complement the results of Wong (2014).

Finally, we examine the effect of hedging on the firm's optimal production decision. When financial hedging by means of trading its output forward is not possible, the firm uses operational hedging to simultaneously manage its exposure to the price risk and that to the background risk. We derive necessary and sufficient conditions under which the firm optimally produces less (more) when the firm is prohibited from trading its output forward. In the special case wherein the background risk is independent of the price risk, we show that the opportunity to hedge induces the risk-averse firm to raise its optimal output level if the predetermined forward price is not below the expected price, thereby rendering the well-known result of Holthausen (1979) that hedging has positive effect on output.

Given that the price risk is positively expectation dependent on the background risk, we show that the firm that is correlation averse (correlation loving) and cross-prudent (cross-imprudent) in the background risk has incentives to produce less (more) when the firm is prohibited from trading its output forward. Correlation aversion (correlation loving) implies that the firm finds it painful to face positive (negative) exposure to the residual price risk in the presence of the background risk. The correlation-averse (correlation-loving) firm as such is induced to produce less (more) than the optimal level when hedging is allowed, leading to a reduction in the firm's profit. Crossprudence (Cross-imprudence) in the background risk implies that the firm finds it more painful to face positive (negative) exposure to the residual price risk in the presence of the background risk when the firm suffers a sure loss. Since the firm earns less when its output is deviated from the optimal level when hedging is allowed, cross-prudence (cross-imprudence) in the background risk further strengthens the firm's incentive to reduce (raise) its output when the firm is prohibited from trading its output forward.

\footnotetext{
5 Both correlation aversion and correlation loving are supported by the empirical evidence in the literature on health economics (Finkelstein et al. 2009). Viscusi and Evans (1990) and Sloan et al. (1998) find support for correlation loving in the case of major injuries, while Evans and Viscusi (1991) find support for correlation aversion in the case of minor injuries (see also Edwards 2008).
} 
The rest of this paper is organized as follows. Section 2 delineates the model of the competitive firm under correlated price and background risks. The firm can hedge the price risk by trading its output forward in the presence of the background risk. Section 3 examines the firm's optimal production and hedging decisions. The separation theorem is shown to hold and the optimality conditions for an over-hedge (nuder-hedge) are characterized. Section 4 examines how hedging affects the firm's optimal production decision. Necessary and sufficient conditions under which hedging has positive (negative) effect on output are derived. Section 5 concludes.

\section{The model}

Consider the competitive firm under price uncertainty à la Sandmo (1971). There is one period with two dates, 0 and 1 . The firm produces a single commodity according to a deterministic cost function, $c(q)$, where $q \geq 0$ is the output level endogenously chosen by the firm at date 0 and $c(q)$ is compounded to date 1 . We assume that the cost function, $c(q)$, satisfies that $c(0)=c^{\prime}(0)=0$, and $c^{\prime}(q)>0$ and $c^{\prime \prime}(q)>0$ for all $q>0 .^{6}$

At date 1 , the firm sells its output in entirety at the prevailing per-unit price, $\tilde{p}$, which is a positive random variable not known ex ante. ${ }^{7}$ Besides the price risk, $\tilde{p}$, the firm faces other sources of risk that are aggregated into a single random variable, $\tilde{z}$. We refer to $\tilde{z}$ as the background risk that can be either financial (risky investment income or random fixed costs) or non-financial (political risk or environmental risk).

The two risks, $\tilde{p}$ and $\tilde{z}$, are jointly distributed according to a known cumulative distribution function (cdf), $F(p, z)$, over support $[p, \bar{p}] \times[\underline{z}, \bar{z}]$, where $0<p<\bar{p}$ and $0<\underline{z}<\bar{z}$. We use $f(p, z)$ to denote the probability density function (pdf) of $\tilde{p}$ and $\tilde{z}$. Let $\mathrm{E}[\cdot]$ be the expectation operator with respect to $F(p, z)$ and $\mathrm{E}[\cdot \mid A]$ be the corresponding expectation operator conditional on the occurrence of event $A$. We denote the marginal cdf and pdf of $\tilde{p}$ by $F_{p}(p)$ and $f_{p}(p)$, respectively. Similar notation applies to $\tilde{z}$.

While the background risk, $\tilde{z}$, is neither hedgeable nor insurable, the firm can hedge the price risk, $\tilde{p}$, by selling (purchasing if negative) $h$ units of its output forward at a predetermined forward price, $p^{f} \in(\underline{p}, \bar{p})$, per unit at date 0 . The firm's profit at date 1 is given by

$$
\pi(\tilde{p})=\tilde{p} q-c(q)+\left(p^{f}-\tilde{p}\right) h
$$

where $\left(p^{f}-\tilde{p}\right) h$ is the gain (loss if negative) from forward trading. We refer to $h$ as the firm's forward position, which is said to be an under-hedge, a full-hedge, or an over-hedge, depending on whether $h$ is smaller than, equal to, or greater than the firm's output level, $q$, respectively.

The firm possesses a bivariate von Neumann-Morgenstern utility function, $u(\pi, z)$, defined over its profit at date $1, \pi$, and the realization of the background risk, $z$.

\footnotetext{
6 The strict convexity of $c(q)$ is driven by the firm's production technology that exhibits decreasing returns to scale.

7 Throughout the paper, random variables have a tilde $(\sim)$ while their realizations do not.
} 
We assume that $u(\pi, z)$ is increasing in each argument, i.e., $u^{(1,0)}(\pi, z)>0$ and $u^{(0,1)}(\pi, z)>0$, and concave in $\pi$, i.e., $u^{(2,0)}(\pi, z)<0$, so that the firm is risk averse in profit, where $u^{(i, j)}(\pi, z)=\partial^{i+j} u(\pi, z) / \partial \pi^{i} \partial z^{j}$.

We say that the firm is correlation averse (correlation loving) if $u^{(1,1)}(\pi, z)<(>) 0$ and cross-prudent (cross-imprudent) in the background risk if $u^{(2,1)}(\pi, z)>(<) 0$ (Eeckhoudt et al. 2007). The former implies that the two risks, $\tilde{p}$ and $\tilde{z}$, behave like substitutes (complements), while the latter implies that a larger realization of the background risk mitigates (aggravates) the detrimental effect of the price risk. As shown by Jokung (2011), correlation aversion is equivalent to the preferences for the 50-50 lottery, [ $\left.\left(\pi_{1}, z_{2}\right) ;\left(\pi_{2}, z_{1}\right)\right]$, over the other 50-50 lottery, [( $\left.\left.\pi_{1}, z_{1}\right) ;\left(\pi_{2}, z_{2}\right)\right]$, whereas correlation loving is equivalent to the opposite preferences, whenever $\pi_{1}<\pi_{2}$ and $z_{1}<z_{2}$. Jokung (2011) shows further that cross-prudence in the background risk is equivalent to the preferences for the 50-50 lottery, $\left[\left(\pi_{1}, z\right) ;\left(\pi_{2}+\tilde{\varepsilon}, z\right)\right]$, over the other 50-50 lottery, $\left[\left(\pi_{1}+\tilde{\varepsilon}, z\right) ;\left(\pi_{2}, z\right)\right]$, whereas cross-imprudence in the background risk is equivalent to the opposite preferences, whenever $\pi_{1}<\pi_{2}$ and $\tilde{\varepsilon}$ is a zero-mean random variable.

The firm's ex-ante decision problem is to choose an output level, $q \geq 0$, and a forward position, $h$, so as to maximize the expected utility of its profit at date 1 :

$$
\max _{q \geq 0, h} \int_{\underline{p}}^{\bar{p}} \int_{\underline{z}}^{\bar{z}} u(\pi(p), z) f(p, z) \mathrm{d} z \mathrm{~d} p
$$

where $\pi(p)$ is given by Eq. (1). The first-order conditions for program (2) are given by

$$
\int_{\underline{p}}^{\bar{p}} \int_{\underline{z}}^{\bar{z}} u^{(1,0)}\left(\pi^{*}(p), z\right)\left[p-c^{\prime}\left(q^{*}\right)\right] f(p, z) \mathrm{d} z \mathrm{~d} p=0
$$

and

$$
\int_{\underline{p}}^{\bar{p}} \int_{\underline{z}}^{\bar{z}} u^{(1,0)}\left(\pi^{*}(p), z\right)\left(p^{f}-p\right) f(p, z) \mathrm{d} z \mathrm{~d} p=0,
$$

where an asterisk $\left(^{*}\right)$ signifies the optimal level. The second-order conditions for program (2) are satisfied given the assumed properties of $u(\pi, z)$ and $c(q)$.

\section{Optimal production and hedging decisions}

Adding Eq. (3) to Eq. (4) yields

$$
\int_{\underline{p}}^{\bar{p}} \int_{\underline{z}}^{\bar{z}} u^{(1,0)}\left(\pi^{*}(p), z\right)\left[p^{f}-c^{\prime}\left(q^{*}\right)\right] f(p, z) \mathrm{d} z \mathrm{~d} p=0 .
$$


It follows from $u^{(1,0)}(\pi, z)>0$ that Eq. (5) reduces to $c^{\prime}\left(q^{*}\right)=p^{f}$, thereby invoking our first proposition.

Proposition 1 Given that the competitive firm can trade its output forward at the predetermined forward price, $p^{f}$, the firm's optimal output level, $q^{*}$, is the one that equates the marginal cost of production, $c^{\prime}\left(q^{*}\right)$, to $p^{f}$.

The intuition for Proposition 1 is as follows. Since the firm can always sell the last unit of its output forward at the predetermined forward price, $p^{f}$, the usual optimality condition applies in that the marginal cost of production, $c^{\prime}\left(q^{*}\right)$, must equal to the known marginal revenue, $p^{f}$, which determines the optimal output level, $q^{*}$. This result holds irrespective of whether the background risk (financial or non-financial) prevails or not.

An immediate implication of Proposition 1 is that the firm's optimal production decision depends neither on the firm's attitude toward risk nor on the incident to the underlying uncertainty. Proposition 1 as such extends the separation theorem of Danthine (1978), Feder et al. (1980), and Holthausen (1979) to the general case of correlated price and background risks.

Evaluating the left-hand side of Eq. (4) at $h=q^{*}$ yields

$$
\int_{\underline{p}}^{\bar{p}} \int_{\underline{z}}^{\bar{z}} u^{(1,0)}\left(p^{f} q^{*}-c\left(q^{*}\right), z\right)\left(p^{f}-p\right) f(p, z) \mathrm{d} z \mathrm{~d} p .
$$

It follows from the second-order conditions for program (2) that $h^{*}>(<) q^{*}$ if, and only if, expression (6) is positive (negative). We state and prove the following proposition.

Proposition 2 Given that the competitive firm can trade its output forward at the predetermined forward price, $p^{f}$, the firm's optimal forward position, $h^{*}$, is an overhedge (under-hedge), i.e., $h^{*}>(<) q^{*}$, if, and only if, the following condition holds:

$$
p^{f}>(<) \mathrm{E}[\tilde{p}]+\int_{\underline{z}}^{\bar{z}} \frac{u^{(1,1)}\left(p^{f} q^{*}-c\left(q^{*}\right), z\right)}{\mathrm{E}\left[u^{(1,0)}\left(p^{f} q^{*}-c\left(q^{*}\right), \tilde{z}\right)\right]}\{\mathrm{E}[\tilde{p}]-\mathrm{E}[\tilde{p} \mid \tilde{z} \leq z]\} F_{z}(z) \mathrm{d} z .
$$

Proof See "Appendix A."

To see the intuition for Proposition 2, consider first the special case wherein $\tilde{p}$ and $\tilde{z}$ are independent. In this special case, we have $\mathrm{E}[\tilde{p} \mid \tilde{z} \leq z]=\mathrm{E}[\tilde{p}]$ for all $z \in[\underline{z}, \bar{z}]$ so that condition $(7)$ reduces to $p^{f}>(<) \mathrm{E}[\tilde{p}]$. Indeed, we can conduct our analysis using the derived utility function, $\hat{u}(\pi)=\mathrm{E}[u(\pi, \tilde{z})]$, thereby resuming the well-known results of Holthausen (1979) that the firm deviates from a full-hedge and opts for an over-hedge (under-hedge) because of the speculative motive driven by $p^{f}>(<) \mathrm{E}[\tilde{p}]$.

In the general case that $\tilde{p}$ and $\tilde{z}$ are correlated, we adopt the following notion of bivariate dependence proposed by Wright (1987). 
Definition 1 The price risk, $\tilde{p}$, is said to be positively (negatively) expectation dependent on the background risk, $\tilde{z}$, if

$$
\mathrm{E}[\tilde{p}] \geq(\leq) \mathrm{E}[\tilde{p} \mid \tilde{z} \leq z],
$$

for all $z \in[\underline{z}, \bar{z}]$, where the inequality is strict for some non-degenerate intervals.

Condition (8) implies that the expectation of $\tilde{p}$ is revised downward (upward) whenever one discovers that $\tilde{z}$ is small, in the precise sense that one is given the truncation that $\tilde{z} \leq z$. According to Lehmann (1966), we can write the covariance between $\tilde{p}$ and $\tilde{z}$ in terms of the cdfs, $F(p, z), F_{p}(p)$, and $F_{z}(z)$ :

$$
\begin{aligned}
\operatorname{Cov}[\tilde{p}, \tilde{z}] & =\int_{\underline{p}}^{\bar{p}} \int_{\underline{z}}^{\bar{z}}\left[F(p, z)-F_{p}(p) F_{z}(z)\right] \mathrm{d} z \mathrm{~d} p \\
& =\int_{\underline{p}}^{\bar{p}} \int_{\underline{z}}^{\bar{z}}\left[F(p \mid \tilde{z} \leq z)-F_{p}(p)\right] F_{z}(z) \mathrm{d} z \mathrm{~d} p \\
& =\int_{\underline{z}}^{\bar{z}}\{\mathrm{E}[\tilde{p}]-\mathrm{E}[\tilde{p} \mid \tilde{z} \leq z]\} F_{z}(z) \mathrm{d} z,
\end{aligned}
$$

where $\operatorname{Cov}[\cdot, \cdot]$ is the covariance operator with respect to $F(p, z)$, and $F(p \mid \tilde{z} \leq z)=$ $F(p, z) / F_{z}(z)$ for all $(p, z) \in[p, \bar{p}] \times[z, \bar{z}]$ is the cdf of $\tilde{p}$ conditional on $\tilde{z} \leq z$. It is evident from Eq. (9) that $\overline{\operatorname{Cov}}[\tilde{p}, \tilde{z}]>(<) 0$ if condition (8) holds. Wright (1987) and Li (2011) show that two random variables that are positively (negatively) correlated need not be positively (negatively) expectation dependent, thereby rendering positive (negative) expectation dependence to be stronger than positive (negative) correlation. ${ }^{8}$ Furthermore, Wright $(1987)$ shows that $\operatorname{Cov}[\tilde{p}, \psi(\tilde{z})]>(<) 0$ for every increasing function, $\psi(\cdot)$, for which the covariance exists if, and only if, $\tilde{p}$ is positively (negatively) expectation dependent on $\tilde{z}$.

Following Eeckhoudt and Schlesinger (2006), we define the utility premium as follows:

$$
\phi_{u}(\pi, \alpha)=\mathrm{E}[u(\pi, \tilde{z})]-\mathrm{E}[u(\pi+\alpha\{\tilde{p}-\mathrm{E}[\tilde{p}]\}, \tilde{z})]
$$

In the following lemma, we derive sufficient conditions under which $\phi_{u}(\pi, \alpha)>0$ so that it measures the "pain" associated with facing exposure, $\alpha$, to the residual price risk, $\tilde{p}-\mathrm{E}[\tilde{p}]$, in the presence of the background risk, $\tilde{z} .{ }^{9}$

Lemma 1 Given that the price risk, $\tilde{p}$, is positively expectation dependent on the background risk, $\tilde{z}$, the utility premium, $\phi_{u}(\pi, \alpha)$, is positive for all $\alpha>(<) 0$ if the bivariate utility function, $u(\pi, z)$, exhibits correlation aversion (correlation loving), i.e., $u^{(1,1)}(\pi, z)<(>) 0$.

\footnotetext{
8 Expectation dependence is, however, the weakest among all extant stronger notions of bivariate dependence than correlation (Chiu 2020; Wong 2021). See Wright (1987) and Li (2011) for an exposition of the alternative notions of bivariate dependence.

${ }^{9}$ Given that $\tilde{p}$ is negatively expectation dependent on $\tilde{z}, \phi_{u}(\pi, \alpha)>0$ for all $\alpha>(<) 0$ if $u^{(1,1)}(\pi, z)>$ $(<) 0$.
} 
Proof Note that

$$
\left.\frac{\partial \mathrm{E}[u(\pi+\alpha\{\tilde{p}-\mathrm{E}[\tilde{p}]\}, \tilde{z})]}{\partial \alpha}\right|_{\alpha=0}=\operatorname{Cov}\left[u^{(1,0)}(\pi, \tilde{z}), \tilde{p}\right]<(>) 0
$$

where the inequality follows from the fact that $\tilde{p}$ is positively expectation dependent on $\tilde{z}$ and $u^{(1,1)}(\pi, z)<(>) 0$ (Wright 1987). Note also that

$$
\frac{\partial^{2} \mathrm{E}[u(\pi+\alpha\{\tilde{p}-\mathrm{E}[\tilde{p}]\}, \tilde{z})]}{\partial \alpha^{2}}=\mathrm{E}\left[u^{(2,0)}(\pi+\alpha\{\tilde{p}-\mathrm{E}[\tilde{p}]\}, \tilde{z})\{\tilde{p}-\mathrm{E}[\tilde{p}]\}^{2}\right]<0
$$

for all $\alpha$. Hence, Eqs. (11) and (12) imply that

$$
\frac{\partial \mathrm{E}[u(\pi+\alpha\{\tilde{p}-\mathrm{E}[\tilde{p}]\}, \tilde{z})]}{\partial \alpha}<(>) 0,
$$

for all $\alpha \geq(\leq) 0$. It then follows from Eq. (10) that $\phi_{u}(\pi, \alpha)>0$ for all $\alpha>(<) 0$.

Lemma 1 shows that the firm that is correlation averse (correlation loving) dislikes positive (negative) exposure to the residual price risk in the presence of the background risk given that $\tilde{p}$ is positively expectation dependent on $\tilde{z}$. Hence, there is an additional hedging motive that induces the firm to sell more (less) of its output forward than a fullhedge so as to reduce the correlation risk. This additional hedging motive reinforces the speculative motive (Holthausen 1979) when $p^{f} \geq(\leq) \mathrm{E}[\tilde{p}]$ so that an overhedge (under-hedge), i.e., $h^{*}>(<) q^{*}$, is optimal. On the other hand, this additional hedging motive counteracts the speculative motive when $p^{f}<(>) \mathrm{E}[\tilde{p}]$ so that the firm's optimal forward position can be either an over-hedge, a full-hedge, or an underhedge, depending on the sign of condition (7). ${ }^{10}$ We as such establish the following proposition.

Proposition 3 Given that the competitive firm can trade its output forward at the predetermined forward price, $p^{f}$, and that the price risk, $\tilde{p}$, is positively expectation dependent on the background risk, $\tilde{z}$, the firm's optimal forward position, $h^{*}$, is an overhedge (under-hedge), i.e., $h^{*}>(<) q^{*}$, if the firm is correlation averse (correlation loving) and $p^{f} \geq(\leq) \mathrm{E}[\tilde{p}]$.

Wong (2014) focuses on the case that the background risk is financial so that $u(\pi, z)$ is reduced to a univariate utility function, $v(\pi+z)$. In this case, correlation aversion, i.e., $u^{(1,1)}(\pi, z)<0$, is equivalent to risk aversion, i.e., $v^{\prime \prime}(\pi+z)<0$. Given that $\tilde{p}$ is positively expectation dependent on $\tilde{z}$, Wong (2014) shows that an over-hedge, i.e., $h^{*}>q^{*}$, is optimal when the forward price is unbiased, i.e., $p^{f}=\mathrm{E}[\tilde{p}]$. Proposition 3 shows that Wong's (2014) result remains valid in the case that the background risk

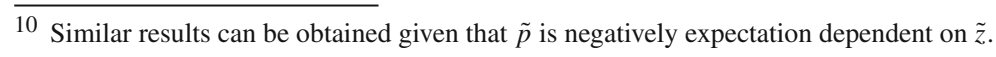


is non-financial only under correlation aversion. In contrast, if the firm is correlation loving, an under-hedge, i.e., $h^{*}<q^{*}$, becomes optimal. This suggests a way to detect whether the firm's preferences exhibit correlation aversion or correlation loving by examining whether the firm's optimal forward position is an over-hedge or an under-hedge, respectively, given that the forward price is unbiased and $\tilde{p}$ is positively expectation dependent on $\tilde{z}$.

\section{The effect of hedging on output}

In this section, we examine the effect of hedging on the firm's optimal production decision. To this end, we consider a benchmark wherein the firm is prohibited from trading its output forward, i.e., $h \equiv 0$. In this benchmark case, the firm's optimal output level, $q^{\circ}$, is uniquely determined by the following first-order condition:

$$
\int_{\underline{p}}^{\bar{p}} \int_{\underline{z}}^{\bar{z}} u^{(1,0)}\left(p q^{\circ}-c\left(q^{\circ}\right), z\right)\left[p-c^{\prime}\left(q^{\circ}\right)\right] f(p, z) \mathrm{d} z \mathrm{~d} p=0 .
$$

It then follows from Eq. (13) and the second-order condition that $q^{*}>(<) q^{\circ}$ if, and only if, the following condition holds:

$$
\int_{\underline{p}}^{\bar{p}} \int_{\underline{z}}^{\bar{z}} u^{(1,0)}\left(p q^{*}-c\left(q^{*}\right), z\right)\left(p-p^{f}\right) f(p, z) \mathrm{d} z \mathrm{~d} p<(>) 0,
$$

where we have used $c^{\prime}\left(q^{*}\right)=p^{f}$ from Proposition 1 .

Expanding the left-hand side of condition (14) by using integration by parts yields the following proposition.

Proposition 4 Prohibiting the competitive firm from trading its output forward at the predetermined forward price, $p^{f}$, induces the firm to optimally produce less (more), i.e., $q^{\circ}<(>) q^{*}$, if, and only if, the following condition holds:

$$
\begin{gathered}
\mathrm{E}\left[u^{(1,0)}\left(\tilde{p} q^{*}-c\left(q^{*}\right), \tilde{z}\right)\right]\left\{\mathrm{E}[\tilde{p}]-p^{f}\right\} \\
+\int_{\underline{p}}^{\bar{p}} u^{(2,0)}\left(p q^{*}-c\left(q^{*}\right), \bar{z}\right) q^{*}\{\mathrm{E}[\tilde{p}]-\mathrm{E}[\tilde{p} \mid \tilde{p} \leq p]\} F_{p}(p) \mathrm{d} p \\
+\int_{\underline{z}}^{\bar{z}} u^{(1,1)}\left(\bar{p} q^{*}-c\left(q^{*}\right), z\right)\{\mathrm{E}[\tilde{p}]-\mathrm{E}[\tilde{p} \mid \tilde{z} \leq z]\} F_{z}(z) \mathrm{d} z \\
-\int_{\underline{p}}^{\bar{p}} \int_{\underline{z}}^{\bar{z}} u^{(2,1)}\left(p q^{*}-c\left(q^{*}\right), z\right) q^{*}\{\mathrm{E}[\tilde{p}]-\mathrm{E}[\tilde{p} \mid \tilde{p} \leq p, \tilde{z} \leq z]\} F(p, z) \mathrm{d} z \mathrm{~d} p<(>) 0 .
\end{gathered}
$$

Proof See "Appendix B." 
A special case of interest is the one wherein $\tilde{p}$ and $\tilde{z}$ are independent. In this special case, we have $F(p, z)=F_{p}(p) F_{z}(z)$ for all $(p, z) \in[p, \bar{p}] \times[\underline{z}, \bar{z}]$ so that $\mathrm{E}[\tilde{p} \mid \tilde{z} \leq z]=\mathrm{E}[\tilde{p}]$ for all $z \in[\underline{z}, \bar{z}]$, and $\mathrm{E}[\tilde{p} \mid \tilde{p} \leq p, \tilde{z} \leq z]=\mathrm{E}[\tilde{p} \mid \tilde{p} \leq p]$ for all $(p, z) \in[\underline{p}, \bar{p}] \times[\underline{z}, \bar{z}]$. Condition $(15)$ becomes

$$
p^{f}>(<) \mathrm{E}[\tilde{p}]+\int_{\underline{p}}^{\bar{p}} \frac{\mathrm{E}\left[u^{(2,0)}\left(p q^{*}-c\left(q^{*}\right), \tilde{z}\right)\right]}{\mathrm{E}\left[u^{(1,0)}\left(\tilde{p} q^{*}-c\left(q^{*}\right), \tilde{z}\right)\right]} q^{*}\{\mathrm{E}[\tilde{p}]-\mathrm{E}[\tilde{p} \mid \tilde{p} \leq p]\} F_{p}(p) \mathrm{d} p
$$

Since $\mathrm{E}[\tilde{p}] \geq \mathrm{E}[\tilde{p} \mid \tilde{p} \leq p]$ for all $p \in[p, \bar{p}]$, the second term on the right-hand side of condition (16) is negative. It then follows from condition (16) that the risk-averse firm optimally produces less when it is prohibited from trading its output forward if the predetermined forward price, $p^{f}$, is not below $\mathrm{E}[\tilde{p}]$. Indeed, we can conduct our analysis using the derived utility function, $\hat{u}(\pi)=\mathrm{E}[u(\pi, \tilde{z})]$, in this special case, thereby resuming the well-known result of Holthausen (1979) that hedging has positive effect on output when $p^{f} \geq \mathrm{E}[\tilde{p}]$.

We now go back to the general case that $\tilde{p}$ and $\tilde{z}$ are correlated. The first term on the left-hand side of condition (15) is negative or positive, depending on whether $p^{f}$ is larger or smaller than $\mathrm{E}[\tilde{p}]$, respectively. The second term is unambiguously negative, which captures the aversion to the price risk that induces the risk-averse firm to produce less when hedging is not possible. The sign of the third term depends on the sign of $\mathrm{E}[\tilde{p}]-\mathrm{E}[\tilde{p} \mid \tilde{z} \leq z]$ and that of $u^{(1,1)}(\pi, z)$. Given that $\tilde{p}$ is positively expectation dependent on $\tilde{z}$ in the sense of Wright (1987), this term is positive (negative) if the firm is correlation averse (correlation loving). The sign of the last term depends on the sign of $\mathrm{E}[\tilde{p}]-\mathrm{E}[\tilde{p} \mid \tilde{p} \leq p, \tilde{z} \leq z]$ and that of $u^{(2,1)}(\pi, z)$. Note that

$$
\begin{gathered}
\mathrm{E}[\tilde{p} \mid \tilde{z} \leq z]-\mathrm{E}[\tilde{p} \mid \tilde{p} \leq p, \tilde{z} \leq z] \\
=\bar{p}-\int_{\underline{p}}^{\bar{p}} \frac{F(x, z)}{F_{z}(z)} \mathrm{d} x-\left[p-\int_{\underline{p}}^{p} \frac{F(x, z)}{F(p, z)} \mathrm{d} x\right] \\
=\int_{p}^{\bar{p}}[1-F(x \mid \tilde{z} \leq z)] \mathrm{d} x+\left[\frac{1-F(p \mid \tilde{z} \leq z)}{F(p \mid \tilde{z} \leq z)}\right] \int_{\underline{p}}^{p} F(x \mid \tilde{z} \leq z) \mathrm{d} x \geq 0 .
\end{gathered}
$$

Given that $\tilde{p}$ is positively expectation dependent on $\tilde{z}$, it follows from Eq. (17) that $\mathrm{E}[\tilde{p}] \geq \mathrm{E}[\tilde{p} \mid \tilde{z} \leq z] \geq \mathrm{E}[\tilde{p} \mid \tilde{p} \leq p, \tilde{z} \leq z]$ for all $(p, z) \in[\underline{p}, \bar{p}] \times[z, \bar{z}]$. Hence, the last term is positive (negative) if the firm is cross-prudent (cross-imprudent) in the background risk. We as such establish the following proposition.

Proposition 5 Given that the price risk, $\tilde{p}$, is positively expectation dependent on the background risk, $\tilde{z}$, and that the predetermined forward price, $p^{f}$, satisfies that 


$$
p^{f} \geq(\leq) \mathrm{E}[\tilde{p}]+\int_{\underline{p}}^{\bar{p}} \frac{u^{(2,0)}\left(p q^{*}-c\left(q^{*}\right), \bar{z}\right)}{\mathrm{E}\left[u^{(1,0)}\left(\tilde{p} q^{*}-c\left(q^{*}\right), \tilde{z}\right)\right]} q^{*}\{\mathrm{E}[\tilde{p}]-\mathrm{E}[\tilde{p} \mid \tilde{p} \leq p]\} F_{p}(p) \mathrm{d} p
$$

prohibiting the competitive firm from trading its output forward induces the firm to optimally produce less (more), i.e., $q^{\circ}<(>) q^{*}$, if the firm is correlation averse (correlation loving) and cross-prudent (cross-imprudent) in the background risk.

When financial hedging by means of trading its output forward is not possible, the firm uses operational hedging to simultaneously manage its exposure to the price risk and that to the background risk. Condition (18) ensures that the predetermined forward price is high (low) enough not to (more than) offset the negative effect due to risk aversion. Given that $\tilde{p}$ is positively expectation dependent on $\tilde{z}$, it follows from Lemma 1 that the utility premium is positive for positive (negative) exposure to the residual price risk in the presence of the background risk if the firm is correlation averse (correlation loving). Hence, correlation aversion (correlation loving) reinforces the net effect due to the predetermined forward price and risk aversion and induces the firm to produce less (more) than $q^{*}$. Since $q^{*}$ maximizes $p^{f} q-c(q)$, the firm's profit is reduced when its output is deviated from $q^{*}$.

Kimball $(1990,1993)$ refers to prudence as preferences for bearing a zero-mean risk in the wealthier states of nature. The prudence utility premium, introduced by Crainich and Eeckhoudt (2008), measures the increase in pain of facing a zero-mean risk in the presence of a sure loss, $\ell>0$. We as such define the prudence utility premium as follows:

$$
\phi_{p}(\pi, \alpha)=\phi_{u}(\pi-\ell, \alpha)-\phi_{u}(\pi, \alpha),
$$

where $\phi_{u}(\pi, \alpha)$ is defined in Eq. (10) and $\ell>0$. Differentiating Eq. (10) with respect to $\pi$ yields

$$
\begin{gathered}
\frac{\partial \phi_{u}(\pi, \alpha)}{\partial \pi}=\mathrm{E}\left[u^{(1,0)}(\pi, \tilde{z})\right]-\mathrm{E}\left[u^{(1,0)}(\pi+\alpha\{\tilde{p}-\mathrm{E}[\tilde{p}]\}, \tilde{z})\right] \\
\approx-\mathrm{E}\left[u^{(2,0)}(\pi, \tilde{z}) \alpha\{\tilde{p}-\mathrm{E}[\tilde{p}]\}\right]=-\alpha \operatorname{Cov}\left[u^{(2,0)}(\pi, \tilde{z}), \tilde{p}\right],
\end{gathered}
$$

where the approximation follows from applying first-order Taylor expansions to $u^{(1,0)}(\pi+\alpha\{p-\mathrm{E}[\tilde{p}]\}, z)$ around $p=\mathrm{E}[\tilde{p}]$. Given that $\tilde{p}$ is positively expectation dependent on $\tilde{z}$, Eq. (20) implies that $\partial \phi_{u}(\pi, \alpha) / \partial \pi<0$ if $u^{(2,1)}(\pi, z)>(<) 0$ and $\alpha>(<) 0$ (Wright 1987). It then follows from Eq. (19) that $\phi_{p}(\pi, \alpha)>0$, which measures the additional pain associated with facing positive (negative) exposure to the residual price risk in the presence of the background risk when the firm that is cross-prudent (cross-imprudent) in the background risk suffers a sure loss, $\ell>0$. Since the firm earns less when its output is deviated from $q^{*}$, the prudence utility premium is positive for positive (negative) exposure to the residual price risk in the 
presence of the background risk if the firm is cross-prudent (cross-imprudent) in the background risk. Hence, cross-prudence (cross-imprudence) in the background risk further strengthens the firm's incentive to reduce (raise) its output, thereby rendering that $q^{\circ}<(>) q^{*}$.

\section{Conclusion}

In this paper, we follow Wong (2014) to examine the competitive firm of Sandmo (1971) that has to make its production and hedging decisions under correlated price and background risks. Unlike Wong (2014), the background risk can be either financial (risky investment income or random fixed costs) or non-financial (political risk or environmental risk), which is accommodated by using a bivariate utility function. We show that the separation theorem holds in that the firm's optimal output level depends neither on the firm's bivariate utility function nor on the joint distribution of the price and background risks. We derive necessary and sufficient conditions under which the firm optimally opts for an over-hedge (under-hedge). We further derive necessary and sufficient conditions under which hedging has positive (negative) effect on the firm's optimal output level. These conditions are shown to be related to the concept of expectation dependence (Wright 1987) and bivariate preferences that include correlation aversion (correlation loving) and cross-prudence (cross-imprudence) à la Eeckhoudt et al. (2007).

While we focus on the behavior of the competitive firm of Sandmo (1971) facing correlated price and background risks, the approach advanced in this paper is completely general. Since multiple sources of uncertainty are ubiquitous, our analysis should be applicable to a broad class of choice problems (e.g., portfolio choice, precautionary saving, and insurance policy). We leave these for future research.

\section{Appendix A: Proof of Proposition 2}

Using integration by parts, we have

$$
\begin{aligned}
& \int_{\underline{p}}^{\bar{p}} \int_{\underline{z}}^{\bar{z}} u^{(1,0)}\left(p^{f} q^{*}-c\left(q^{*}\right), z\right) p f(p, z) \mathrm{d} p \mathrm{~d} z \\
& =\int_{\underline{p}}^{\bar{p}} u^{(1,0)}\left(p^{f} q^{*}-c\left(q^{*}\right), \bar{z}\right) p \int_{\underline{z}}^{\bar{z}} f(p, z) \mathrm{d} z \mathrm{~d} p \\
& \quad-\int_{\underline{p}}^{\bar{p}} \int_{\underline{z}}^{\bar{z}} u^{(1,1)}\left(p^{f} q^{*}-c\left(q^{*}\right), z\right) p \int_{\underline{z}}^{z} f(p, y) \mathrm{d} y \mathrm{~d} z \mathrm{~d} p \\
& =u^{(1,0)}\left(p^{f} q^{*}-c\left(q^{*}\right), \bar{z}\right) \bar{p} \int_{\underline{p}}^{\bar{p}} \int_{\underline{z}}^{\bar{z}} f(p, z) \mathrm{d} p \mathrm{~d} z \\
& -\int_{\underline{p}}^{\bar{p}} u^{(1,0)}\left(p^{f} q^{*}-c\left(q^{*}\right), \bar{z}\right) \int_{\underline{p}}^{p} \int_{\underline{z}}^{\bar{z}} f(x, z) \mathrm{d} z \mathrm{~d} x \mathrm{~d} p
\end{aligned}
$$




$$
\begin{aligned}
& -\int_{\underline{z}}^{\bar{z}} u^{(1,1)}\left(p^{f} q^{*}-c\left(q^{*}\right), z\right) \bar{p} \int_{\underline{p}}^{\bar{p}} \int_{\underline{z}}^{z} f(p, y) \mathrm{d} y \mathrm{~d} p \mathrm{~d} z \\
& +\int_{\underline{p}}^{\bar{p}} \int_{\underline{z}}^{\bar{z}} u^{(1,1)}\left(p^{f} q^{*}-c\left(q^{*}\right), z\right) \int_{\underline{p}}^{p} \int_{\underline{z}}^{z} f(x, y) \mathrm{d} x \mathrm{~d} y \mathrm{~d} p \mathrm{~d} z \\
& =u^{(1,0)}\left(p^{f} q^{*}-c\left(q^{*}\right), \bar{z}\right) \bar{p}-\int_{\underline{p}}^{\bar{p}} u^{(1,0)}\left(p^{f} q^{*}-c\left(q^{*}\right), \bar{z}\right) F_{p}(p) \mathrm{d} p \\
& -\int_{\underline{z}}^{\bar{z}} u^{(1,1)}\left(p^{f} q^{*}-c\left(q^{*}\right), z\right) \bar{p} F_{z}(z) \mathrm{d} z \\
& +\int_{\underline{p}}^{\bar{p}} \int_{\underline{z}}^{\bar{z}} u^{(1,1)}\left(p^{f} q^{*}-c\left(q^{*}\right), z\right) F(p, z) \mathrm{d} p \mathrm{~d} z \\
& =u^{(1,0)}\left(p^{f} q^{*}-c\left(q^{*}\right), \bar{z}\right) \mathrm{E}[\tilde{p}]-\int_{\underline{z}}^{\bar{z}} u^{(1,1)}\left(p^{f} q^{*}-c\left(q^{*}\right), z\right) F_{z}(z) \mathrm{d} z \mathrm{E}[\tilde{p}] \\
& +\int_{\underline{p}}^{\bar{p}} \int_{\underline{z}}^{\bar{z}} u^{(1,1)}\left(p^{f} q^{*}-c\left(q^{*}\right), z\right)\left[F(p, z)-F_{p}(p) F_{z}(z)\right] \mathrm{d} p \mathrm{~d} z \\
& =\mathrm{E}\left[u^{(1,0)}\left(p^{f} q^{*}-c\left(q^{*}\right), \tilde{z}\right)\right] \mathrm{E}[\tilde{p}] \\
& +\int_{\underline{z}}^{\bar{z}} u^{(1,1)}\left(p^{f} q^{*}-c\left(q^{*}\right), z\right)\{\mathrm{E}[\tilde{p}]-\mathrm{E}[\tilde{p} \mid \tilde{z} \leq z]\} F_{z}(z) \mathrm{d} z,
\end{aligned}
$$

where the second last equality follows from $\mathrm{E}[\tilde{p}]=\bar{p}-\int_{\underline{p}}^{\bar{p}} F_{p}(p) \mathrm{d} p$. Substituting Eq. (A.1) into expression (6) yields condition (7).

\section{Appendix B: Proof of Proposition 4}

Using integration by parts, we have

$$
\begin{aligned}
& \int_{\underline{p}}^{\bar{p}} \int_{\underline{z}}^{\bar{z}} u^{(1,0)}\left(p q^{*}-c\left(q^{*}\right), z\right) p f(p, z) \mathrm{d} z \mathrm{~d} p \\
& =\int_{\underline{p}}^{\bar{p}} u^{(1,0)}\left(p q^{*}-c\left(q^{*}\right), \bar{z}\right) p \int_{\underline{z}}^{\bar{z}} f(p, z) \mathrm{d} z \mathrm{~d} p \\
& \quad-\int_{\underline{p}}^{\bar{p}} \int_{\underline{z}}^{\bar{z}} u^{(1,1)}\left(p q^{*}-c\left(q^{*}\right), z\right) p \int_{\underline{z}}^{z} f(p, y) \mathrm{d} y \mathrm{~d} z \mathrm{~d} p \\
& =u^{(1,0)}\left(\bar{p} q^{*}-c\left(q^{*}\right), \bar{z}\right) \bar{p} \int_{\underline{p}}^{\bar{p}} \int_{\underline{z}}^{\bar{z}} f(p, z) \mathrm{d} z \mathrm{~d} p \\
& -\int_{\underline{p}}^{\bar{p}}\left[u^{(1,0)}\left(p q^{*}-c\left(q^{*}\right), \bar{z}\right)\right.
\end{aligned}
$$




$$
\begin{aligned}
& \left.+u^{(2,0)}\left(p q^{*}-c\left(q^{*}\right), \bar{z}\right) p q^{*}\right] \int_{\underline{z}}^{\bar{z}} \int_{\underline{p}}^{p} f(x, z) \mathrm{d} x \mathrm{~d} z \mathrm{~d} p \\
& -\int_{\underline{z}}^{\bar{z}} u^{(1,1)}\left(\bar{p} q^{*}-c\left(q^{*}\right), z\right) \bar{p} \int_{\underline{p}}^{\bar{p}} \int_{\underline{z}}^{z} f(p, y) \mathrm{d} y \mathrm{~d} p \mathrm{~d} z \\
& +\int_{\underline{p}}^{\bar{p}} \int_{\underline{z}}^{\bar{z}}\left[u^{(1,1)}\left(p q^{*}-c\left(q^{*}\right), z\right)\right. \\
& \left.+u^{(2,1)}\left(p q^{*}-c\left(q^{*}\right), z\right) p q^{*}\right] \int_{\underline{p}}^{p} \int_{\underline{z}}^{z} f(x, y) \mathrm{d} y \mathrm{~d} x \mathrm{~d} z \mathrm{~d} p \\
& =u^{(1,0)}\left(\bar{p} q^{*}-c\left(q^{*}\right), \bar{z}\right) \bar{p}-\int_{\underline{z}}^{\bar{z}} u^{(1,1)}\left(\bar{p} q^{*}-c\left(q^{*}\right), z\right) \bar{p} F_{z}(z) \mathrm{d} z \\
& -\int_{\underline{p}}^{\bar{p}}\left[u^{(1,0)}\left(p q^{*}-c\left(q^{*}\right), \bar{z}\right)+u^{(2,0)}\left(p q^{*}-c\left(q^{*}\right), \bar{z}\right) p q^{*}\right] F_{p}(p) \mathrm{d} p \\
& +\int_{\underline{p}}^{\bar{p}} \int_{\underline{z}}^{\bar{z}}\left[u^{(1,1)}\left(p q^{*}-c\left(q^{*}\right), z\right)+u^{(2,1)}\left(p q^{*}-c\left(q^{*}\right), z\right) p q^{*}\right] F(p, z) \mathrm{d} z \mathrm{~d} p .
\end{aligned}
$$

Using integration by parts, we have

$$
\begin{gathered}
\int_{\underline{p}}^{\bar{p}} u^{(1,0)}\left(p q^{*}-c\left(q^{*}\right), \bar{z}\right) F_{p}(p) \mathrm{d} p \\
=u^{(1,0)}\left(\bar{p} q^{*}-c\left(q^{*}\right), \bar{z}\right) \int_{\underline{p}}^{\bar{p}} F_{p}(p) \mathrm{d} p \\
-\int_{\underline{p}}^{\bar{p}} u^{(2,0)}\left(p q^{*}-c\left(q^{*}\right), \bar{z}\right) q^{*} \int_{\underline{p}}^{p} F_{p}(x) \mathrm{d} x \mathrm{~d} p \\
=u^{(1,0)}\left(\bar{p} q^{*}-c\left(q^{*}\right), \bar{z}\right)\{\bar{p}-\mathrm{E}[\tilde{p}]\} \\
-\int_{\underline{p}}^{\bar{p}} u^{(2,0)}\left(p q^{*}-c\left(q^{*}\right), \bar{z}\right) q^{*}\{p-\mathrm{E}[\tilde{p} \mid \tilde{p} \leq p]\} F_{p}(p) \mathrm{d} p .
\end{gathered}
$$

Using integration by parts, we have

$$
\begin{aligned}
& \int_{\underline{p}}^{\bar{p}} \int_{\underline{z}}^{\bar{z}} u^{(1,1)}\left(p q^{*}-c\left(q^{*}\right), z\right) F(p, z) \mathrm{d} z \mathrm{~d} p \\
& \quad=\int_{\underline{z}}^{\bar{z}} u^{(1,1)}\left(\bar{p} q^{*}-c\left(q^{*}\right), z\right) \int_{\underline{p}}^{\bar{p}} F(p, z) \mathrm{d} p \mathrm{~d} z
\end{aligned}
$$




$$
\begin{gathered}
-\int_{\underline{p}}^{\bar{p}} \int_{\underline{z}}^{\bar{z}} u^{(2,1)}\left(p q^{*}-c\left(q^{*}\right), z\right) q^{*} \int_{\underline{p}}^{p} F(x, z) \mathrm{d} x \mathrm{~d} z \mathrm{~d} p \\
=\int_{\underline{z}}^{\bar{z}} u^{(1,1)}\left(\bar{p} q^{*}-c\left(q^{*}\right), z\right)\{\bar{p}-\mathrm{E}[\tilde{p} \mid \tilde{z} \leq z]\} F_{z}(z) \mathrm{d} z \\
-\int_{\underline{p}}^{\bar{p}} \int_{\underline{z}}^{\bar{z}} u^{(2,1)}\left(p q^{*}-c\left(q^{*}\right), z\right) q^{*}\{p-\mathrm{E}[\tilde{p} \mid \tilde{p} \leq p, \tilde{z} \leq z]\} F(p, z) \mathrm{d} z \mathrm{~d} p .
\end{gathered}
$$

Substituting Eqs. (A.3) and (A.4) into Eq. (A.2) yields

$$
\begin{aligned}
& \int_{\underline{p}}^{\bar{p}} \int_{\underline{z}}^{\bar{z}} u^{(1,0)}\left(p q^{*}-c\left(q^{*}\right), z\right) p f(p, z) \mathrm{d} z \mathrm{~d} p \\
& =u^{(1,0)}\left(\bar{p} q^{*}-c\left(q^{*}\right), \bar{z}\right) \mathrm{E}[\tilde{p}]-\int_{\underline{p}}^{\bar{p}} u^{(2,0)}\left(p q^{*}-c\left(q^{*}\right), \bar{z}\right) q^{*} \mathrm{E}[\tilde{p} \mid \tilde{p} \leq p] F_{p}(p) \mathrm{d} p \\
& \quad-\int_{\underline{z}}^{\bar{z}} u^{(1,1)}\left(\bar{p} q^{*}-c\left(q^{*}\right), z\right) \mathrm{E}[\tilde{p} \mid \tilde{z} \leq z] F_{z}(z) \mathrm{d} z \\
& \quad+\int_{\underline{p}}^{\bar{p}} \int_{\underline{z}}^{\bar{z}} u^{(2,1)}\left(p q^{*}-c\left(q^{*}\right), z\right) q^{*} \mathrm{E}[\tilde{p} \mid \tilde{p} \leq p, \tilde{z} \leq z] F(p, z) \mathrm{d} z \mathrm{~d} p . \quad \text { (A.5) }
\end{aligned}
$$

Furthermore, we have

$$
\begin{aligned}
& \int_{\underline{p}}^{\bar{p}} \int_{\underline{z}}^{\bar{z}} u^{(1,0)}\left(p q^{*}-c\left(q^{*}\right), z\right) f(p, z) \mathrm{d} z \mathrm{~d} p \\
& =u^{(1,0)}\left(\bar{p} q^{*}-c\left(q^{*}\right), \bar{z}\right)-\int_{\underline{p}}^{\bar{p}} u^{(2,0)}\left(p q^{*}-c\left(q^{*}\right), \bar{z}\right) q^{*} F_{p}(p) \mathrm{d} p \\
& \quad-\int_{\underline{z}}^{\bar{z}} u^{(1,1)}\left(\bar{p} q^{*}-c\left(q^{*}\right), z\right) F_{z}(z) \mathrm{d} z \\
& \quad+\int_{\underline{p}}^{\bar{p}} \int_{\underline{z}}^{\bar{z}} u^{(2,1)}\left(p q^{*}-c\left(q^{*}\right), z\right) q^{*} F(p, z) \mathrm{d} z \mathrm{~d} p .
\end{aligned}
$$

Substituting Eqs. (A.5) and (A.6) into condition (14) yields condition (15).

\section{References}

Chiu, W.H.: Financial risk taking in the presence of correlated non-financial background risk. J. Math. Econ. 88, 167-179 (2020)

Crainich, D., Eeckhoudt, L.: On the intensity of downside risk aversion. J. Risk Uncertan. 36, 267-276 (2008) 
Danthine, J.-P.: Information, futures prices, and stabilizing speculation. J. Econ. Theory 17, 79-98 (1978)

Edwards, R.D.: Health risk and portfolio choice. J. Bus. Econ. Stat. 26, 472-485 (2008)

Eeckhoudt, L., Rey, B., Schlesinger, H.: A good sign for multivariate risk taking. Manage. Sci. 53, 117-124 (2007)

Eeckhoudt, L., Schlesinger, H.: Putting risk in its proper place. Am. Econ. Rev. 96, 280-289 (2006)

Evans, W.N., Viscusi, W.K.: Estimation of state-dependent utility functions using survey data. Rev. Econ. Stat. 73, 94-104 (1991)

Feder, G., Just, R.E., Schmitz, A.: Futures markets and the theory of the firm under price uncertainty. Quarterly J. Econ. 94, 317-328 (1980)

Finkelstein, A., Luttmer, E.F.P., Notowidigdo, M.J.: Approaches to estimating the health state dependence of the utility function. Am. Econ. Rev. 99, 116-121 (2009)

Hassan, T.A., Hollander, S., van Lent, L., Tahoun, A.: Firm-level political risk: measurement and effects. Quarterly J. Econ. 134, 2135-2202 (2019)

Holthausen, D.M.: Hedging and the competitive firm under price uncertainty. Am. Econ. Rev. 69, 989-995 (1979)

Jokung, O.: Risk apportionment via bivariate stochastic dominance. J. Math. Econ. 47, 448-452 (2011)

Kimball, M.S.: Precautionary saving in the small and in the large. Econometrica 58, 53-73 (1990)

Kimball, M.S.: Standard risk aversion. Econometrica 61, 589-611 (1993)

Lehmann, E.L.: Some concepts of dependence. Ann. Math. Stat. 37, 1137-1153 (1966)

$\mathrm{Li}$, J.: The demand for a risky asset in the presence of a background risk. J. Econ. Theory 146, 372-391 (2011)

Lu, H., Oh, W.-Y., Kleffner, A., Chang, Y.K.: How do investors value corporate social responsibility? Market valuation and the firm specific contexts. J. Bus. Res. 125, 14-25 (2021)

Mossin, J.: Aspects of rational insurance purchasing. J. Polit. Econ. 76, 553-568 (1968)

Sandmo, A.: On the theory of the competitive firm under price uncertainty. Am. Econ. Rev. 61, 65-73 (1971)

Sloan, F.A., Viscusi, W.K., Chesson, H.W., Conover, C.J., Whetten-Goldstein, K.: Alternative approaches to valuing intangible health losses: the evidence for multiple sclerosis. J. Health Econ. 17, 475-497 (1998)

Viscusi, W.K., Evans, W.N.: Utility functions that depend on health status: estimates and economic implications. Am. Econ. Rev. 80, 353-374 (1990)

Wong, K.P.: Hedging and the competitive firm under correlated price and background risk. Decis. Econ. Finance 37, 329-340 (2014)

Wong, K.P.: Comparative risk aversion with two risks. J. Math. Econ. (2021) (in press)

Wright, R.: Expectation dependence of random variables, with an application in portfolio theory. Theory Decis. 22, 111-124 (1987)

Publisher's Note Springer Nature remains neutral with regard to jurisdictional claims in published maps and institutional affiliations. 